\title{
Meet The Flow Chemist - Dr. Amol A. Kulkarni
}

\section{Timothy Noël ${ }^{1}$}

Published online: 7 September 2020

(C) Akadémiai Kiadó 2020

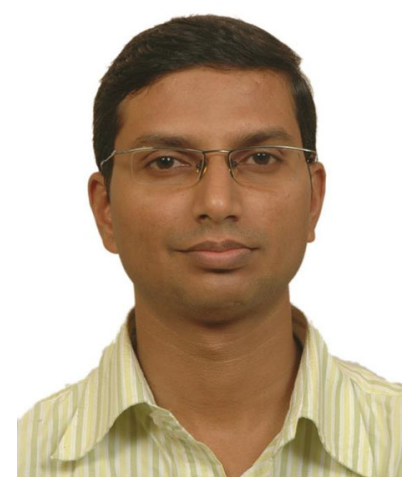

\begin{tabular}{ll}
\hline Name & Dr. Amol A. Kulkarni \\
Date of Birth & December 3 ${ }^{\text {rd }}, 1976$ \\
Position & Senior Principal Scientist, Chemical Engineering \\
& \& Process Development Division, \\
& CSIR-National Chemical Laboratory, \\
& Pune - 411008, India \\
E-mail & aa.kulkarni@ @ ncl.res.in \\
Homepage & http://academic.ncl.res.in/aa.kulkarni \\
Education & B. Chem. Eng. (1998), M. Chem. Eng. (2000), \\
& Ph.D. (2003) \\
Awards & VASVIK award for 'Chemical Sciences \& \\
& Technology' (2016) \\
& Swarna Jayanti Fellowship (2015) by Dept. of \\
Sci. \& Tech. (GoI) & IUSSTF Research Fellowship (2010) at \\
& Massachusetts Institute of Technology (MIT, \\
Cambridge, USA) \\
• Young Scientist Awards: INSA (2009), INAE \\
(2009), Indian Academy of Sciences (2011), \\
CSIR (2011), OPPI (2015) \\
• Humboldt Research Fellowship (2004) Max \\
Planck Inst. for Dynamics of Complex \\
Technical Systems (Magdeburg, Germany) \\
\hline
\end{tabular}

1) When did you start with flow chemistry? Describe the first paper or the first experiments.

My first opportunity to learn and do a continuous flow synthesis happened at the Max Planck Institute-Magdeburg in 2004 with Prof. Achim Kienle when I was asked to intensify a homogeneous and heterogeneous catalytic reaction for synthesis of butyl acetate, which was to be further extended for continuous heterogeneous catalytic reactive distillation. I had designed a compact catalytic reactor where we could make a stack of plates that helped us achieve flow synthesis of the desired ester. It was great to see that I was just able to change the flow rates to change the outlet composition. Could perform a large number of experiments in a very short time, which was not possible in conventional mode.

2) What are the main benefits of flow that convinced you to use and implement this technology in your research?

Timothy Noël

T.Noel@tue.nl

1 Department of Chemical Engineering and Chemistry, Eindhoven University of Technology, Eindhoven, Netherlands

Being a chemical engineer, I look at flow synthesis little differently. During any synthesis, batch or flow, several phenomena viz. mixing, heat transfer, mass transfer, reaction happen simultaneously and they all govern the overall rate of reaction. Importantly, each of these phenomena have a characteristic time scale and the slowest phenomenon needs more attention to intensify a process, provided the choice of conditions do not affect the selectivity. Nature of energy source (viz. kinetic energy, pressure energy, ultrasound, microwave, photons, etc.) as well as the energy consumption decide the design of the system as well as the overall performance. Eventually, the flow chemistry community needs to ensure that the efforts put in developing a proof of the concept is actually a realizable process. Secondly, compactness of the systems brings out several advantages as well as challenges in space utilization/optimization. In my research, we focus on quantifying these phenomena and use them to propose new scalable designs. 
3) What do you think the future holds for flow chemistry?

Future of flow chemistry is amazing. The early efforts in miniaturization have helped the community to explore unthinkable flash chemistry. It opens a window to perform new transformations as finally chemistry is equivalent to creativity. I strongly believe that flow chemistry should become a routine synthesis tool so that the focus can be shifted to efforts on developing new concepts that were not possible before. The field is expanding rapidly but one may not spend too much time on realizing a versatile synthesis platform that doesn't remain affordable for a new entrant in the field. Simple setups need pumps, tubes, fittings, valves, BPRs, thermostats and monitoring cum control unit. A lot can be done using these to begin with. Work-up after reactions is still a time-consuming job and it varies from reactions to reaction, so there is a lot of space in working on how you avoid it or minimize it.

4) Do you have any relevant tips for newcomers in the field?

My tips for newcomers in the field are: (i) be open for collaboration and be ready to adapt new domains or people with expertise in electronics, automation, modelling and simulations, design, robotics, mechatronics, digitization of chemistry etc., complex and big problems needs more minds to work together and give credit to everyone who makes it work, (ii) creativity is a human thing, flow chemistry will help you explore it very neatly in any areas of your interest whether it is in polymers, materials, catalysis, biotransformation or organometallic chemistry, and (iii) there is no age barrier that makes one rethink on whether to enter in this field, in fact the most experienced chemists and chemical engineers can make the best contributions to the field.

\section{My three most relevant papers related to flow chemistry:}

1. Sharma, B. M.; Atapalkar, R. S. and Kulkarni, A. A. (2019) Continuous flow solvent free organic synthesis involving solids (reactants/products) using screw reactor, Green Chemistry, 2019, 21, 5639 - 5646

Here we demonstrated for the first time that several types of reactions can be carried out using a single platform with minimal or almost no solvent thereby showing sold-phase reactions as a possible component that can even be smoothly incorporated in multistep flow synthesis. This is just the beginning, as it opens new challenges like how to do such reactions if one of the byproducts from reactions is a gas or when to do synthesis in vertical and horizontal orientation of the screw reactors.

2. Vasudevan, N.; Sharma, M. K.; Reddy, D. S. and Kulkarni, A. A. (2018) A multistep continuous flow synthesis of cystic fibrosis medicine Ivacaftor, Reaction Chemistry \& Engineering, 3 (4), 520-526

This was an interesting case, where for the first time we demonstrated continuous flow synthesis of the world's most expensive drug. The fine collaboration and team effort between synthetic organic chemists and chemical engineers can actually take-up a complex synthesis challenge.

3. Sharma, M. K.; Potdar, S. B And Kulkarni, A. A. (2017) Pinched Tube Flow Reactor: Hydrodynamics and Suitability for Exothermic Multiphase Reactions, AICHE J., 63(1), 358-365

This is more of a flow reactor design and this amazing concept of pinching of simple tubes has helped us develop the concept and demonstrate it in a big way by industrial implementations. The design has been licensed to Amar Equipment Pvt. Ltd. (Mumbai) and they have designed and supplied pinched tube reactors from $100 \mathrm{ml}$ volume to $165 \mathrm{~L}$, the largest one is run at a throughput of $5000 \mathrm{~L} / \mathrm{hr}$. 


\section{Meet the Flow Chemist - Dr. Richard Bourne}

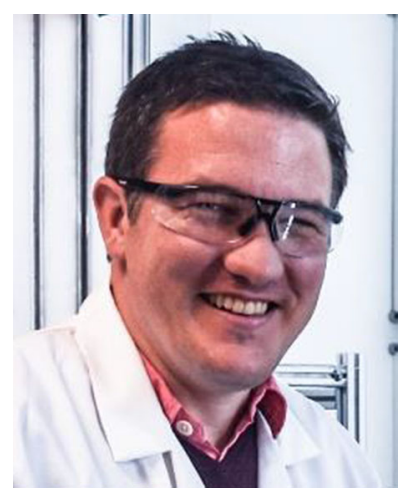

1) When did you start with flow chemistry? Describe the first paper or the first experiments.

I performed my first flow chemistry experiment as a master's student in the group of Sir Martyn Poliakoff. I was working alongside a PDRA (Dr. Jason Hyde) and was performing experiments using supercritical carbon dioxide at 100 bar. As a naive master student, I really hadn't realised what I was letting myself in for. The chemists in the group looked more like mechanics - wrenches and spanners were everywhere! The lab also hummed with activity, carbon dioxide was spurting out of back pressure regulators, sample valves were triggering and hydrogen maximators (compressors) were providing a chorus! Ever since I have really enjoyed the vibrant nature of flow chemistry labs and the development of new technologies.

2) What are the main benefits of flow that convinced you to use and implement this technology in your research?

My independent research has focused on the automated development of pharmaceutical processes. Whilst we have observed the same advantages in terms of safety and facile scale-up that many groups have reported, I believe that one of the critical advantages is the enhanced reproducibility of reactions performed in flow systems. The high quality of results (especially when combined with automation/robotics) gives much better confidence in engineering a scaled-up system compared to colleagues that are using classical round bottom flasks. This advantage can be further exploited by using intensified conditions, to enable tracking of undesired reactions with greater accuracy and thus providing far better models than simple atmospheric pressure batch chemistry ever could.

3) What do you think the future holds for flow chemistry?

I hope that the next generation of chemists are trained early about the advantages of flow chemistry and that every

\begin{tabular}{ll}
\hline Name & Richard Bourne \\
Date of Birth & $7^{\text {th }}$ December 1981 \\
Position & Associate Professor, University of Leeds (UK) \\
E-mail & R.A.Bourne@ leeds.ac.uk \\
Homepage & www.bournelab.co.uk \\
Education & PhD - Chemistry - University of \\
& Nottingham (UK) \\
& Advisor: Sir Martyn Poliakoff CBE FRS \\
& MChem - Chemistry (hons) - University of \\
& Nottingham (UK) \\
Awards & Senior Research Fellow of the Royal Academy \\
& of Engineering \\
\hline
\end{tabular}

undergraduate degree includes a lab practical that uses a flow reactor. I believe that in the next 10 years, flow chemistry will evolve to the point that automated chemical server farms will operate as a service and be able to take requests from around the world to run chemistry remotely in centralized locations with high-end online analysis. This centralized model of performing chemistry will possibly result in huge waste reduction and lower costs enabling much larger libraries for active ingredient discovery and potentially revolutionize the next generation of chemical products. But they'll always be challenging chemistries that a robot can never perform!

4) Do you have any relevant tips for newcomers in the field?

Commercial flow systems are generally excellent at performing lab flow chemistry and capable of doing experiments over a wide range of chemical space. But I highly recommend that newcomers also try to build components, or even their whole own flow system. The freedom that constructing parts of your own platform can result in very high impact publications and capabilities not yet commercially available. I've particularly enjoyed recent work such as that by Milad Abolhasani using, innovative online reaction monitoring systems and Tim Noël using photochemical flow coiled reactors.

\section{My three most relevant papers related to flow chemistry:}

1) Adam D. Clayton, Artur M. Schweidtmann, Graeme Clemens, Jamie A. Manson, Connor J. Taylor, Carlos G. Niño, Thomas W. Chamberlain, Nikil Kapur, A. John Blacker, Alexei A. Lapkin and Richard A. Bourne, Automated self-optimisation of multi-step reaction and separation processes using machine learning, Chemical Engineering Journal, 384, (2020), 123440

This paper was a fantastic team effort combining automated reactor platforms with multistage chemical platforms. The 
use of self-optimising systems that include multiple reactor stages and/or work up are increasingly growing and the team really valued using new technologies such as the Freactor miniature CSTRs and Zaiput membrane separators.

2) Artur M. Schweidtmann, Adam D. Clayton, Nicholas Holmes, Eric Bradford, Richard A. Bourne and Alexei A. Lapkin, Machine learning meets continuous flow chemistry: Automated optimization towards the Pareto front of multiple objectives, Chemical Engineering Journal, 352, (2019), 277.

This paper was a real highlight and demonstrated the value of collaborating with other disciplines. In this work we applied a new machine learning algorithm for optimisation of multiple objectives and demonstrating the importance of trade-offs between economic and environmental factors when generating new flow processes.
3) Nicholas Holmes, Geoffrey R. Akien, A. John Blacker, Robert L. Woodward, Rebecca E. Meadows and Richard A. Bourne, Self-optimisation of the final stage in the synthesis of EGFR kinase inhibitor AZD9291 using an automated flow reactor, Reaction Chemistry and Engineering, 1, (2016). 366

This paper was my groups first attempt at developing a self-optimising system for development of a flow process in the synthesis of a pharmaceutical compound. Watching the system autonomously run this optimisation and send updates during the night was incredibly exciting.

Publisher's note Springer Nature remains neutral with regard to jurisdictional claims in published maps and institutional affiliations. 